\title{
LA CANCELACIÓN DE LA GUERRA Y LOS CAMINOS DE LA PAZ \\ Teresa Santiago*
}

De acuerdo con una famosa sentencia de Kant en el segundo apartado definitivo de Hacia la paz perpetua (1795), la paz consiste en un conjunto de condiciones jurídicas, políticas y morales que tienen que ser construidas pues, de suyo, no se dan de manera 'natural' en la sociedad:

El estado de paz entre hombres que viven juntos no es un estado de naturaleza (status naturalis), que es más bien un estado de guerra, es decir, un estado en el que, si bien las hostilidades no se han declarado, sí existe una constante amenaza de que se declaren. El estado de paz debe, por tanto, ser instaurado, pues la omisión de hostilidades no es todavía garantía de paz. ${ }^{1}$ litana-I.

* Universidad Autónoma Metropo-

${ }^{1}$ Hacia la paz perpetua, 1999, Madrid, Biblioteca Nueva, Clásicos del pensamiento, p. 81 .
Son varias las ideas contenidas en el texto anterior y que vale la pena considerar. En primer lugar está la tesis de acuerdo con la cual el estado natural de los hombres es, en el sentido hobbesiano, el de la confrontación permanente. La guerra no necesita ser efectiva, basta con que exista la 'constante amenaza', para formarnos el panorama de que las relaciones humanas no son naturalmente armoniosas sino que, por el contrario, se caracterizan por la tensión, la confrontación y el disenso. A diferencia de Hobbes, Kant no discute ni especula -por lo menos en este texto- sobre cuáles podrían ser las causas que llevan a los hombres a enfrentarse constantemente. ${ }^{2}$

${ }^{2}$ En otros textos como Idea (s) para una historia universal en clave cosmopolita y Comienzo presunto de la historia humana (ambos de 1786), Kant se ocupa más ampliamente de este tema. En este último distingue tres etapas por las cuales transita el hombre, desde el estado en el cual lo domina su natu- 

NOTAS

De hecho podríamos afirmar que una tesis que poco va a variar a lo largo de su pensamiento es la de que las consecuencias del conflicto (y, por ende, de la guerra) son más importantes que sus causas. Para entrar en conflicto con el prójimo, o bien para decidir la guerra, no se necesitan causas (o razones) muy elaboradas: cualquier motivo puede, en su momento, servir como justificación. En todo caso, lo importante es fijar la atención sobre los resultados que se obtienen de los enfrentamientos; no quién es el vencedor, sino qué gana el grupo, la sociedad o la humanidad entera. Aunque resulte paradójico, los Estados requieren de la experiencia de la guerra, como los hombres requieren del conflicto, para decidirse a abandonar el salvajismo que corresponde al estado de naturaleza. La naturaleza pone el conflicto, la insociable sociabilidad, y el hombre es quien tiene que desactivar ese mecanismo natural una vez que se ha percatado de que por el camino de la guerra es imposible realizar su humanidad, su racionalidad y, por ende, los fines morales de la especie.

Sobre el concepto de insociable sociabilidad, dice Kant:

raleza más tosca, hasta el momento en el cual consigue para sí los elementos indispensables para realizar sus propios fines.
El hombre tiene una tendencia a socializarse, porque en tal estado siente más su condición de hombre. [...] Pero también tiene una fuerte inclinación a individualizarse (aislarse), porque encuentra simultáneamente en sí mismo la insociable cualidad de doblegar todo a su mero capricho [...] pues bien, esta resistencia es aquello que despierta todas las fuerzas del hombre y le hace vencer su inclinación a la pereza, impulsándole por medio de la ambición, el afán de dominio o la codicia, a procurarse una posición entre sus congéneres, a los que no puede soportar, pero de los que tampoco es capaz de prescindir. ${ }^{3}$

\section{Y agrega:}

Así se dan los auténticos primeros pasos desde la barbarie hacia la cultura $[\ldots]$ de este modo van desarrollándose poco a poco todos los talentos, así va formándose el gusto $\mathrm{e}$, incluso, mediante una continua ilustración, comienza a constituirse una manera de pensar que, andando el tiempo, puede transformar su tosca disposición natural hacia el discernimiento ético en principios prácticos $\mathrm{y}$, finalmente, transformar un consenso social urgido patológicamente en un ámbito moral. ${ }^{4}$

${ }^{3}$ Ideas para una historia universal en clave cosmopolita, 1994, Madrid, Tecnos, Clásicos del pensamiento, p. 8.

${ }^{4}$ Idem, p. 9. 
De manera que si careciéramos de esa raíz contradictoria, seríamos seres naturalmente pacíficos, pero igualmente ineficaces en lo tocante a la construcción de las instituciones políticas indispensables para darle una salida racional al conflicto. La primera meta hacia la cual son impulsados los hombres por la sabiduría natural es el contrato fundacional con el cual se pone límite a la libertad salvaje del estado de naturaleza. Se trata del orden civil o estado de derecho. Con este pacto no se inaugura la sociedad, sino la civilidad. Se trata, sin embargo, como ya apuntábamos, de un primer paso en el largo y penoso camino hacia la completa realización política y moral de la especie a la cual identifica Kant con una 'paz perpetua'.

En efecto, la paz perpetua es la última meta política de la especie y la más difícil de alcanzar. Es el nivel en el cual se consolidan órdenes normativos previos. También significa un salto cualitativo en el desarrollo moral de la especie, ya que si bien el orden civil puede ser alcanzado incluso 'por un pueblo de demonios' con tal de que sean racionales, la paz perpetua no puede ser impuesta desde el exterior, tiene que ser el resultado de acatar el veto de la razón que manda abandonar la guerra. Pero esto no sucederá hasta que no haya probado los sinsabores de la guerra pues,

a través de las guerras y sus exagerados e incesantes preparativos, mediante la indigencia que por esta causa ha de acabar experimentando internamente todo Estado incluso en tiempos de paz, la Naturaleza (a los hombres) los arrastra a abandonar el estado anómico propio de los salvajes e ingresar en una confederación de pueblos. ${ }^{5}$

Con la paz perpetua se habría alcanzado el pleno desarrollado de la capacidad racional del ser humano, que no es sólo la de conocer y explicar el mundo, sino la de plantearse cualquier clase de fines. A esta capacidad le llama Kant 'aptitud' para la cultura. ${ }^{6}$ El ser humano es un ser para la cultura porque puede plantearse cualquier clase de fines, entre ellos fines morales y políticos cada vez más altos. En otras palabras, Kant nos estaría invitando a no conformarnos con la paz temporal que es sólo el preludio de nuevas guerras cada vez más cruentas y a aspirar a una paz definitiva.

Podemos pasar ahora a preguntarnos: ¿cómo se construye la paz

${ }^{5}$ Idem, p. 14.

${ }^{6}$ Esta noción es tratada por Kant en la Crítica del juicio, 1961, Buenos Aires, Losada, §83. 
perpetua? Para responder a ella tenemos que abrirnos paso en las principales tesis de Hacia la paz. Antes de iniciar nuestra reflexión, debemos anotar lo siguiente en cuanto a la estructura del texto: está formado por dos grandes apartados, que contienen respectivamente los artículos preliminares y los definitivos. La separación en dos tipos de apartados sugiere algo en lo que es importante reparar; el logro de la paz supone dos momentos claramente diferenciables: el primero consiste en poner límites a la guerra a través de ciertas reglas o leyes, con el fin de reducir el espacio a la legitimación del recurso bélico, mientras que el segundo es el que corresponde, en realidad, a la construcción de la paz. Vamos a considerar primero los artículos preliminares. Los puntos a los que se refieren son los siguientes:

1. La prohibición de los tratados de paz sobre la reserva de una guerra futura.

2. La adquisición de un Estado por otro a través de la herencia, el intercambio, la compra o la donación.

3. La desaparición paulatina de los ejércitos.

4. Las guerras no deben servir para contraer deudas públicas.

5. Debe prohibirse la intervención del Estado en los asuntos internos o la constitución de otro.
6. Deben prohibirse las hostilidades de tipo tal que hagan imposible 'la confianza mutua en la paz futura'.

Las prohibiciones contenidas en 1,5 y 6 son consideradas por Kant 'estrictas', esto es, leyes que no pueden no cumplirse, mientras que 2, 3 y 4 son leyes 'laxas' pues su cumplimiento puede depender de las circunstancias del momento. Interesa resaltar sobre todo que el objetivo del conjunto formado por ambos grupos de leyes es establecer, a pesar de las guerras, las condiciones mínimas para el entendimiento entre los Estados y no se haga imposible la paz futura. Así, esa base mínima es de orden jurídico y también moral porque pretende no sólo poner límites a la guerra, sino establecer condiciones de confianza mutua. También es importante que ninguno de los artículos hace mención a un derecho de guerra (ius ad bellum). Lo que revela el espíritu pacifista del tratado, que se expresa muy claramente en los artículos 3 y 5. Por contraste, hallamos prohibiciones para la conducta de guerra (in bello), artículo 6, así como para después de la guerra (post bellum), artículos 1, 2 y 4 .

Un tema que merece atención especial es el principio de no intervención (artículo 5), objeto de 
múltiples discusiones. De acuerdo con Kant: "Ningún Estado debe inmiscuirse por la fuerza en la constitución y en el gobierno de otro", esto es, no hay razón que justifique el intervenir en los asuntos internos de otro Estado pues se le considera una persona moral y, por ende, no puede ser tomado como una cosa que pudiera ser objeto de la ambición o el abuso de otro que es su igual. No obstante, Kant considera la situación en la cual, a causa de pugnas internas, un Estado se divide en dos (o más) grupos encontrados que llevan a la desaparición del orden civil, con lo cual se legitima el que una fuerza exterior intervenga a solicitud de alguna de las partes para restaurar dicho orden. Esta observación no debe interpretarse como una excepción al principio. En el caso referido, no existe ya el Estado; lo que impera es la anarquía y por ello cualquiera de las partes tiene el derecho de solicitar ayuda para restablecer el orden fracturado.

No podemos pasar de largo el hecho de que Kant no haya considerado otro tipo de circunstancias en las cuales podría cuestionarse el principio de no intervención. Es el caso de lo que hoy se conoce como 'ayuda humanitaria' y, por supuesto, el caso en el cual se violan los dere-

${ }^{7}$ Hacia la paz..., p. 76. chos básicos de los individuos. Ésta podría considerarse una falla en el modo como Kant concibió el artículo 5. Sin embargo, de acuerdo con Luc Langlois, aún se puede rescatar la idea de un derecho de intervención (kantiano) consistente con este tipo de casos,

siempre y cuando estemos de acuerdo en enmendar lo dicho en la teoría jurídica sobre dos puntos [...] centrales que la animan y que no atentan a la coherencia principal. $\mathrm{La}$ primera de estas enmiendas toca la tesis de la inviolabilidad del poder establecido, la segunda, la forma institucional que debe revestir la alianza de pueblos por los que el $j u s$ gentium puede definir un derecho que sea verdaderamente exigible. ${ }^{8}$

${ }^{8}$ Je ferai donc l'hypothèse qu'un droit d'intervention demeure concevable dans une optique kantienne, à condition toute-foi que l'on consente à amender la lettre de la thèorie juridique sur deux points qui, me semble-t-il, peuvent être distingués de l'intuition centrale qui l'anime et qui ne portent pas etteinte à sa cohérence principielle. Le premier de ces amendements touche à la theèse de l'inviolabilité du pouvoir établi [...] le second, à la forme institutionnelle que doit revêrtir l'alliance des peuples pour le jus gentium puisse définir un droit qui soit véritablement exigible, "Kant et la question du droit d'intervention", en Pierre Laberge, Guy Lafrance y Denis Dumas, L'Ánnée 1795. Kant. Essai sur la Paix, 1997, París, Vrin, p. 61. 

NOTAS

Ciertamente, para admitir un derecho de intervención tendríamos que asegurarnos de que no viole la soberanía de los Estados (i.e., que no pase por encima de un poder establecido legítimamente, o bien, que no vaya en contra de su carácter de persona moral) y, en segundo lugar, que sea el producto de la aplicación de una norma perteneciente al derecho de gentes y, por ende, exigible; no el producto de la decisión de uno o varios Estados para favorecer sus intereses.

Pero éstas son condiciones aún muy generales. Aunque establecen una normatividad mínima, no resuelven el problema de cómo proceder respecto de muchos casos en los cuales, por ejemplo, estamos frente a regímenes no democráticos -en la jerga kantiana, no republicanos- $-\mathrm{y}$ que, por consiguiente, no garantizan todos los derechos propios de las democracias, pero en los que no se violan los derechos humanos y las condiciones de vida pueden ser, incluso, favorables para el desarrollo de los ciudadanos. Este caso, el de regímenes que no siendo democracias liberales pueden ser considerados 'decentes' ha sido planteado por Rawls en The Law of People, 1999.

En dicha obra, el recién fallecido filósofo distingue entre cinco diferentes tipos de sociedades domésticas: pueblos liberales razo- nables; pueblos decentes; Estados proscritos; Estados en condiciones desfavorables; ${ }^{9}$ y absolutismos benignos. Como sugieren los términos empleados por Rawls en dicha clasificación, son los Estados proscritos y los Estados en condiciones desfavorables los candidatos a ser objeto del derecho de intervención. La idea que anima el texto de Rawls no parece estar tan alejada de la noción kantiana de 'enemigo injusto' (Metafísica de las costumbres), frente al cual el derecho de un Estado es ilimitado; el enemigo injusto:

es aquél cuya voluntad públicamente expresada (sea de palabra o de obra) denota una máxima según la cual, si se convirtiera en regla universal, sería imposible un estado de paz entre los pueblos y tendría que perpetuarse el estado de naturaleza. ${ }^{10}$

La injusticia del enemigo a la cual alude la definición, radica en

${ }^{9}$ Los Estados proscritos no respetan los derechos humanos y son agresivos respecto de la comunidad internacional; los Estados en condiciones desfavorables no necesariamente practican una conducta agresiva hacia el exterior, pero en la medida en que no han podido desarrollarse adecuadamente (económica y políticamente) pueden no ser muy respetuosos de los derechos humanos.

${ }^{10}$ Metafísica de las costumbres, 1999, Madrid, Tecnos, Clásicos del pensamiento, p. 189. 
que ostenta una conducta agresiva frente a la comunidad de pueblos. No es injusto por las causas que esgrime, sino porque la máxima por la que se guía es contraria a la aspiración de armonía que mueve al resto de los Estados al recurrir a la confrontación para dirimir los conflictos. Frente a un Estado 'infractor' es legítimo el empleo de la fuerza, lo que nos lleva a concluir que Kant no era del todo contrario a la idea de un derecho de intervención tratándose de los Estados que ponen en peligro la posibilidad de formar una comunidad pacífica.

Ahora bien, una vez que Kant ha establecido los artículos preliminares que sirven de antesala al proyecto de la paz perpetua, se da a la tarea de señalar cuáles son las condiciones para que ésta pueda ser instaurada:

i. El establecimiento de una constitución republicana para todo Estado;

ii. Un derecho de gentes fundado en una federación de Estados libres, y,

iii. Un derecho cosmopolita que debe limitarse a las condiciones de 'hospitalidad universal'.

Algo hemos dicho sobre el pacto fundacional que lleva a los hombres a abandonar el estado de naturaleza a favor de un orden civil (i). Una constitución republicana es, a los ojos de Kant, la forma más perfecta de plasmar el pacto que da origen a la sociedad civil.

En cuanto a la federación de Estados libres (ii), en realidad Kant está pensando en una confederación de naciones. De allí su insistencia en que sobre ella no debe existir ningún poder que controle y norme las relaciones entre los distintos Estados. No es casual, por tanto, su observación en el sentido de que dicha federación 'no debería ser un Estado de pueblos'. En efecto, Kant tiene cuidado de mostrar que la idea de una federación de pueblos no se interprete en los términos de la sociedad civil, en la cual los ciudadanos tienen que someterse al poder soberano.

A diferencia de los ciudadanos, los Estados no pueden renunciar a la soberanía que les es propia para someterse a un poder superior. Además, siempre estaría la amenaza de que ese poder se convirtiera en una dictadura mundial que sería aún más lamentable que la propia situación anómica en que se encuentran los Estados antes de formar una comunidad pacífica. La elección racional es, entonces, el federalismo libre, lo que conecta bien con la idea de un derecho de gentes siempre y cuando éste se entienda no como un derecho de guerra, sino como el derecho a formar una coalición pacífica que 
incluya a todo los pueblos del planeta.

La guerra, la más implacable maestra, obliga a los Estados a formar una federación libre. Pero la libertad salvaje y, por ende, el estado de naturaleza en la cual se encuentran las naciones, también se refleja de manera importante en la conducta inhospitalaria hacia los extranjeros. Por ello incluye Kant entre los artículos definitivos de la paz perpetua, un derecho cosmopolita (iii):

Se trata en este artículo, como en los anteriores, de derecho y no de filantropía, y hospitalidad significa aquí el derecho de un extranjero a no ser tratado hostilmente por el hecho de haber llegado al territorio de otros. ${ }^{11}$

Con la introducción del derecho cosmopolita se pretende incluir un nivel normativo a partir del cual se creen condiciones de justicia que respondan a la concepción de persona humana que tiene Kant, en tanto poseedora de dignidad y de autonomía y, así, garantizar el respeto a los derechos fundamentales de todo ser racional.

El ideal cosmopolita kantiano no consiste en borrar las fronteras territoriales del planeta, sino en suprimir las condiciones de injus-

${ }^{11}$ Hacia la paz..., p. 95. ticia y hacer del derecho la norma a la cual pueda recurrir cualquier individuo sin importar el lugar en el que se encuentre. Si el comercio y la cultura se extienden por todos los rincones de la tierra, es deseable que también la justicia se convierta en una noción global. Pensamiento utópico o no, para Kant el proyecto de una paz definitiva no puede ser concebido sin una visión cosmopolita del derecho. Con ello superó no sólo a sus contemporáneos. En particular, debemos reconocer al cosmopolitismo kantiano que hoy en día aparezca en las agendas de las instituciones internacionales el tema de los derechos fundamentales como algo que compete a la humanidad entera.

Podemos sintetizar las ideas rectoras del proyecto kantiano para una paz perpetua de la siguiente forma: se requiere de una base jurídica mínima que ayude a erradicar la desconfianza (natural) entre los Estados, con lo cual queda claro que la paz perpetua no se logra de un día para otro ni equivale a los pactos de paz particulares propios de la dinámica guerra-paz-guerra que pretende superarse.

En cuanto a los propios Estados: un primer paso consistiría en formar repúblicas cuya constitución sea lo más perfecta posible $\mathrm{y}$, sólo entonces, tendría sentido plantear 
la posibilidad de reunirse en una comunidad de iguales cuyo fin sea la paz. Esa paz tiene que garantizar condiciones de justicia para todo sujeto en cualquier parte del planeta, por lo que tiene que darse antes un derecho cosmopolita.

El proyecto satisface, sin duda, las exigencias para transformar las relaciones entre los Estados, pero ¿qué o quién garantiza que se den las condiciones para esa transformación? Kant recurre aquí, como ya lo había hecho en Idea para una historia, a la noción de 'astucia natural', ahora caracterizada:

como la gran artista naturaleza [...] en cuyo curso mecánico destaca ostensiblemente una finalidad: que a través del antagonismo de los hombres surja la armonía, incluso contra su voluntad, razón por la cual se le llama indistintamente destino, como causa necesaria de los efectos producidos según sus leyes, desconocidas para nosotros, o providencia, por referencia a la finalidad del curso del mundo, como la sabiduría profunda de una causa más elevada que se guía por el fin final objetivo del género humano y que predetermina el curso del mundo. ${ }^{12}$

Esta 'causa necesaria' cuyos efectos pueden verse como producidos 'según leyes' tiene como finali-

${ }^{12}$ Idem, p. 100. dad la armonía entre los hombres y, para conseguirlo se vale de la rivalidad, el conflicto y, principalmente, de la guerra. Esta causa eficiente no se descubre mediante el conocimiento, ni tampoco se infiere, sino que se piensa por 'analogía' con la práctica artística. La gran artista naturaleza ha dispuesto no sólo que los hombres puedan vivir bien sino que deban vivir bien. Ése es el destino de la especie. Se trata, en efecto, de un destino moral y no meramente biológico.

Kant ofrece tres tesis sobre el modo como la naturaleza garantiza el sometimiento al deber de construir la paz y así cumplir con el destino moral de la especie. La primera hace referencia, una vez más, a la guerra como aquello que obliga a los hombres a llegar a un pacto para formar el orden civil. No se trata de una unidad basada en el amor fraterno o la solidaridad, sino simple y llanamente de una cuestión de racionalidad práctica, por ello afirma Kant que el problema del establecimiento del Estado "tiene solución incluso para un pueblo de demonios (siempre que tengan entendimiento)" ${ }^{13}$

En segundo lugar, establece la tesis de las diferencias de lenguaje y religión como necesarias para engendrar un estado de conflicto y

${ }^{13}$ Idem, p. 105. 
pugna, pero que al incrementarse la cultura, hará posibles el equilibrio y las coincidencias en la paz, "una paz generada y garantizada mediante el equilibrio de fuerzas en la más viva competencia [...] y no como resultado del quebrantamiento de todas las energías". ${ }^{14}$

Por último y en apoyo de esta tesis, se refiere Kant al espíritu comercial de los pueblos, esto es, a la posibilidad de potenciar esta disposición natural. Opuesto a la guerra, pues supone una mínima base de confianza mutua, el espíritu comercial puede actuar como un mecanismo conducente a propiciar otro tipo de relaciones entre los Estados.

Quizá nos parezca ingenua la confianza de Kant en las bondades de la actividad comercial. Sin embargo, no hay que olvidar que esta tesis va unida a las dos anteriores, esto es, $s i$ se acepta la tesis sobre las condiciones de justicia y de respeto al derecho, no es difícil imaginar entonces una fórmula que regule las relaciones comerciales entre las naciones de manera que no se cometan los abusos frecuentes de las más poderosas hacia las más débiles.

Una última observación sobre el concepto de providencia, se hace indispensable. Se trata de un concepto que pertenece a la familia de

${ }^{14}$ Idem, p.107. los conceptos teleológicos, cuyo uso está ampliamente fundamentado en la Crítica del juicio. En efecto, en esa compleja obra, Kant intentó probar que la tarea de la facultad de juzgar reflexivamente va dirigida a la posibilidad de brindar contenido a las proposiciones teleológicas, esto es, a establecer una 'legalidad' diferente a la mecánica o causal. En este sentido, la proposición kantiana sobre la garantía proveniente de la 'artista naturaleza' para que la especie logre sus fines más altos no puede ser interpretada en el sentido de que esas condiciones son determinantes; esto es, no determinan ningún hecho de la realidad, son ejemplos de condiciones propicias para que la razón práctica se proyecte hacia esos ideales.

Nuestra última pregunta es la siguiente: ¿cuál es el mayor impedimento para la construcción de la paz? La respuesta no deja de ser sorprendente: la supuesta incompatibilidad entre la moral y la política a la que aluden los políticos cuando intentan justificar sus decisiones. Todos quieren realizar las mejores acciones, beneficiar a los ciudadanos y lograr la armonía entre los pueblos, pero pronto 'comprueban' que no es posible guiarse por esos buenos propósitos, y concluyen que la política más eficaz tiene que dejarse guiar por los intereses particulares. Se impone la 
visión del político realista y sagaz para el cual es imposible conciliar moral y política.

Para Kant no hay tal incompatibilidad, en todo caso lo que hay es la incapacidad (o mala fe) del político de ver más allá de sus propios intereses, porque desde la plataforma del poder se adquiere una conveniente miopía moral. El lugar en donde aparecen estas observaciones es el artículo 'secreto' de Hacia la paz. La ironía de Kant no podría ser más sutil: en él recomienda a los filósofos no mezclarse en política y a los políticos no asesorarse de filósofos que pudieran curarlos de su miopía moral.

Ciertamente moral y política son difíciles de compaginar. En la práctica política no es fácil guiarse por el respeto a la ley moral. Kant no intenta negar este hecho. Su crítica va dirigida a la pretensión de que la práctica política deje de aspirar a ser guiada por la ley moral bajo el supuesto de que 'ya se ha visto' que ambas son incompatibles. El pragmatismo político, la sagacidad de que tanto se ufanan quienes detentan el poder, colabora con los mecanismos de la naturaleza para que éstos se impongan sobre cualquier intento de hacer realidad la conciliación entre política y moral.

La manera de disolver la discrepancia entre la moral y la política es por medio del principio de publicidad que reza así: "Todas las máximas que necesitan de la publicidad (para no fracasar en sus propósitos) concuerdan con el derecho y la política a la vez." A este principio tendrían que ser sometidas todas las máximas con las cuales se justifican las decisiones de los políticos. Sin duda serían muy pocas las que podrían pasar la prueba de tal principio. Los ejemplos empleados por Kant se refieren a dos situaciones de la mayor relevancia para los Estados: a) el derecho civil y un supuesto 'derecho' a la rebelión, $y, b$ ) el derecho de gentes (y un supuesto derecho a la guerra). En cuanto al primer caso, Kant ya había presentado argumentos en contra de este 'derecho' ${ }^{15}$ en otros textos. Ahora nos recuerda que para que tal derecho fuera legítimo tendría que pasar la prueba del principio de publicidad, pero es evidente que nadie aceptaría como un derecho algo que va en contra del orden jurídico mismo, a saber, la posibilidad de su disolución. Algo similar sucede con el segundo caso, el de la asociación de los pueblos en una comunidad pacífica. El ideal de la paz es, sin duda, un propósito que puede hacerse público, no así el de la guerra.

${ }^{15}$ Crf., Sobre el tópico: esto podría ser correcto en teoría, pero no vale para la práctica (1793). 

NOTAS

Con el principio de publicidad Kant se anticipa a una idea hoy en día muy discutida en torno a la necesidad de democratizar la política por medio de la creación de una esfera pública en la cual participen los ciudadanos en la discusión y las decisiones que los afectan directamente.

La conclusión que se ajusta mejor al tratado de paz de Kant es el veto de la razón práctica que se expresa así: No debe haber guerra, que se encuentra en la parte final de la exposición del derecho de gentes de la Metafisica de las costumbres. $\mathrm{El}$ argumento del cual se sigue dicho veto es el siguiente: Si no se puede probar que algo es, se puede intentar probar que no es. Pero si no se consigue ninguna de las dos cosas, se puede mantener alguna de ellas con algún propósito práctico o moral para alcanzar un determinado fin, tal que la máxima de proponérselo constituya un deber. A lo que nos obliga la conjetura no es a creer que el fin es realizable, sino a actuar de acuerdo con la idea de ese fin. Por tanto, la idea de la paz definitiva no es una idea vacía mientras se le considere un proyecto al que podemos acercarnos poco a poco y de manera permanente. 